Yang, Rongguang

\section{MAT a set of microarray data manage- ment and analysis tools}

\author{
Rongguang Yang, Aiming Zhang, Aldo Massimi, Thomas M \\ Harris, Susanne Kneitz, Geoffrey Childs \& Raju Kucherlapati \\ Department of M olecular Genetics, Albert Einstein College of M edicine, \\ Bronx, New York, USA
}

DNA Microarrays have become important in genome-wide expression studies. However, to fully use the power of the microarrays, more sophisticated and userfriendly data management and analysis tools have to be developed. We have developed a software package called MAT (Microarray Analysis Tools). MAT consists of a data entry tool, a web-based analysis tool and a relational database. User interfaces written in Java and JDBC are used to access the relational database (Sybase Adaptive server 11.5). The data entry tool is a form-based interface for user-friendly data entry. The web-based analysis tools consist of a synthetic image view tool, a graphic plot tool to display results from a series of experiments and a neural network-based data clustering tool. All of the interfaces are inter-connected. A user can view a single experiment or multiple experiments. A user can plot a time course, as well as a correlation graph with different experimental conditions. The mouse and human clone databases are automatically updated by searching through GenBank. The program also allows for the user to obtain GenBank data for any clone. Finally, the cluster tool will provide an advanced algorithm for clustering selected data. Our MAT tools are facilitating gene expression studies at AECOM, and can be adapted for use in other research laboratories.

\section{Zhang, Li}

\section{New algorithms for microarray data analysis}

\section{Li Zhang}

EGCRC, University of California at San Francisco, San Francisco, California, USA

Microarray technologies are generating new data at a breathtaking speed. Because of the sheer size of these data and the technical complexity involved in microarray chip designs, it is a challenge to assess the reliability and the accuracy of these data. In our research using Affymetrix microarrays, many genes of interest are expressed at low levels and are only slightly up- or down-regulated under various biological conditions. By using the software offered by Affymetrix Inc., these genes are mostly called "absent". However, in the original raw data on the chip images, we have found consistent and reproducible patterns regarding those lowly expressed genes. Based on this finding we designed and tested a new algorithm for expression profile evaluation. When applied to our microarray data set, the new method seems to be able to identify genes that are simply called "absent" by Affymetrix's method and actually have reproducible changes in gene expression in duplicate experiments. Furthermore, compared with Affymetrix's algorithm, the new algorithm reports far fewer genes as differentially expressed when comparing two samples that were prepared under identical biological conditions. Thus, the new method is believed to have less false positives and more true positives in recognising differentially expressed genes, a highly desired property for the microarray data analysis.
Zhao, Lue Ping

\section{Mapping complex traits with single nucleotide polymorphisms}

\author{
Lue Ping Zhao, Corinne Aragaki, Li Hsu \& Filemon Q uiaoit \\ Q uantitative Genetic Epidemiology, Division of Public Health Sciences, Fred \\ Hutchinson Cancer Research Center, 1100 Fairview Ave. N, M W 805, \\ Seattle, Washington, USA
}

Molecular geneticists are developing the third generation human genome map with single nucleotide polymorphisms (SNPs), which can be assayed through chipbased microarrays. One use of these SNP markers is to locate loci that may be responsible for complex traits through linkage/linkage-disequilibrium analysis. We describe a semiparametric method for combined linkage/linkage-disequilibrium analysis using SNP markers. Asymptotic results are obtained for the estimated parameters, and the finite sample properties are evaluated through a simulation study. We also applied this technique to a simulated genome scan experiment for mapping a complex trait with two major genes. This experiment shows that separate linkage and linkage-disequilibrium analyses correctly detected the signals of both major genes, but the rates of false positive signals seem high. Combining linkage and linkage-disequilibrium signals, the analysis yielded much stronger and clearer signals for the presence of two major genes than two separate analyses.

Zhou, Xiaochuan

\section{Light-directed, programmable microarray synthesis}

\author{
Xiaochuan Zhou ${ }^{1}$, Xiaolian Gao' ${ }^{2}$ Eric LeProust ${ }^{2}$, Jean Philippe \\ Peppllois's, Peilin $\mathrm{Yu}^{2}$, Hua Zhang ${ }^{2}$, Erdogan Gulari', \\ O nnop Srivannavit ${ }^{3} \&$ Ning Gulari $^{3}$ \\ ${ }^{1}$ Xeotron Corporation, Houston, Texas 77030, USA \\ ${ }^{2}$ Department of Chemistry, University of Houston, \\ Houston, Texas 77204-5641, USA \\ ${ }^{3}$ Department of Chemical Engineering, University of M ichigan, \\ Ann Arbor, M ichigan 48105, USA
}

A new method of parallel synthesis suitable for making microarrays of biomolecules has been developed. The method involves the use of novel solution chemistry and programmable photolithographic instruments. The chemistry described herein lies in its use of photo-generated reagents to affect otherwise conventional synthesis chemistry. The photolithographic instrument consists of a digital light projector that is capable of generating photolithographic patterns directly from a computer. We will describe our results on the synthesis of oligonucleotides and peptides using photogenerated acids.

Our method of using photo-generated reagents enables light-initiation of conventional synthesis reactions. This new approach takes advantage of well-established synthesis procedures and materials and thus, promises simple, flexible, high quality and low cost parallel synthesis of microarrays of oligonucleotides, peptides and diverse molecular sequences. The use of a digital light projector makes light-directed synthesis flexible and eliminates the need for expensive photomask-based photolithographic equipment and associated cleanroom facilities.

Using the new method as a technological foundation we are currently developing a programmable maskless automated microarray synthesiser that is affordable and technically manageable by most laboratories interested in biochips. The availability of such an instrument would significantly accelerate the processes of gene analysis and the development of gene-based applications. 